\title{
Challenges to estimating contagion effects from observational data
}

\author{
Elizabeth L. Ogburn
}

\section{Background}

\section{Motivating example}

Suppose that students attending the residential Faber College are measured and weighed at the start and close of each school year, and a complete social network census is taken, cataloguing all social ties among members of the student body. In addition, researchers have access to basic demographic covariates measured on each student. Researchers are interested in testing whether there is a contagion effect for body mass index (BMI): if one individual-the ego-gains (or looses) weight, does that make his or her social contacts-the alters-more likely to do the same? They are also interested in estimating the contagion effect if one exists: if an ego gains (or looses) weight, what is the expected increase (or decrease) in the alters' body mass indices?

There are many different procedures one could use to test for or estimate a contagion effect, using different models, different assumptions, different sets of covariates, different ways of calculating intervals or uncertainty, and the list goes on. In order for a procedure to be useful, it has to satisfy two requirements. First, it has to isolate the causal effect of the ego's change in BMI on the alters' changes in BMI from potential other sources of similarity between the ego's and the alters' outcomes. This has to do with confounding, which is the subject of Section 4

The second requirement for a useful analysis is that it must be generalizable to populations beyond the precise student body used in the analysis. We would like to be able to extrapolate what we learn about contagion from the Faber student body to contagion of BMI in similar college populations across different colleges or even across different years at Faber College. Assume that the student body we observe

Elizabeth L. Ogburn

Johns Hopkins Bloomberg School of Public Health, 615 N. Wolfe St. Baltimore MD, e-mail: eogburne jhsph.edu; supportfromONRgrantN000141512343 
at Faber College is representative of these other student populations, that is, that the true underlying contagion effect for the observed sample of Faber students is the same as the true underlying contagion effect in the other college populations to which we want to extrapolate. Then one way to determine whether we are warranted in extrapolating from Faber students to the other similar groups of students is to calculate a confidence interval for the true contagion effect, based on a model of asymptotic growth of the sample. For example, if the sample is large enough that a central limit theorem approximately holds for the contagion effect estimate, then a Gaussian confidence interval around the sample mean is approximately valid. Under the assumption of the same true underlying contagion effect, our confidence that this interval covers the true contagion effect for Faber College students is the same as our confidence that it covers the true contagion effect for students at a different college or in a different year. As in many settings for statistical inference, asymptotics are appropriate not because we care about an infinite population but because they shed light on finite samples. This requires valid statistical inference, which is the subject of Section 5 .

\section{Defining causal effects}

Questions about the influence one subject has on the outcome of another subject are inherently questions about causal effects: contagion is a causal effect on an ego's outcome at time $t$ of his alter's outcome at time $s$ for some $s<t$. Causal effects are defined in terms of potential or counterfactual outcomes (see e.g. Hernán. 2004, Rubin 2005). In general, a unit-level potential outcome, $Y_{i}(z)$, is defined as the outcome that we would have observed for subject $i$ if we could have intervened to set that subject's treatment or exposure $Z_{i}$ to value $z$. A contagion effect of interest for dyadic data might be a contrast of counterfactuals of the form $Y_{\text {ego }}^{t}\left(y_{\text {alter }}^{t-1}\right)$, for example $E\left[Y_{\text {ego }}^{t}(y)-Y_{\text {ego }}^{t}(y-1)\right]$ would be the expected difference in the ego's counterfactual outcome at time $t$ had the alter's outcome at time $t-1$ been set to $y$ compared to $y-1$. In data comprised of independent dyads this contagion effect is well-defined, but social networks represent a paradigmatic opportunity for interference, whereby one subject's exposure may affect not only his own outcome but also the outcomes of his social contacts and possibly other subjects. Under interference, the traditional unit-level potential outcomes are not well-defined. Instead, $Y_{i}(\mathbf{z})$ is the outcome that we would have observed if we could have set the vector of exposures for the entire population, $\mathbf{Z}$, to $\mathbf{z}=\left(z_{1}, \ldots, z_{n}\right)$ where for each $i, z_{i}$ is in the support of $Z$. The causal inference literature distinguishes between interference, which is present when one subject's treatment or exposure may affect others' outcomes, and contagion, which is present when one subject's outcome may influence or transmit to other subjects (e.g. Ogburn and VanderWeele, 2014a), but in fact they are usually intertwined. Consider three Faber students: Alex, Andy, and Ari, all friends with each other. Alex's outcome at time $t$ depends on both Andy's and Ari's outcomes at time $t-1$, Andy's outcome at time $t$ depends on Alex's and Ari's 
at time $t-1$, and Ari's outcome at time $t$ depends on Alex's and Andy's at time $t-1$. This results in a situation that is hardly distinguishable from the hallmarks of interference: $Y_{\text {Alex }}^{t}\left(y_{\text {Andy }}^{t-1}, y_{\text {Ari }}^{t-1}\right), Y_{\text {Andy }}^{t}\left(y_{\text {Alex }}^{t-1}, y_{\text {Ari }}^{t-1}\right)$, and $Y_{\text {Ari }}^{t}\left(y_{\text {Alex }}^{t-1}, y_{\text {Andy }}^{t-1}\right)$ are potential outcomes that depend on multiple "treatments" and those treatments are overlapping across subjects. Furthermore, just as in settings with interference, a counterfactual outcome for node $i$ that omits some of the treatments to which node $i$ is exposed (i.e. the outcomes at time $t-1$ for some of $i$ 's alters) is not well-defined. This has been overlooked in most of the literature on contagion in observational social network data, which generally focuses on alter-ego pairs, thereby inherently considering illdefined counterfactuals like $Y_{\text {Alex }}^{t}\left(y_{\text {Andy }}^{t-1}\right)$.

This points to an under-appreciated challenge for the study of contagion in a social network: simply defining the causal effect of interest. If researchers sample non-overlapping alter-ego dyads from the network then $Y_{\text {ego }}^{t}\left(y_{\text {alter }}^{t-1}\right)$ may be welldefined, but if they wish to use all of the available data, comprised of overlapping dyads, causal effects must be defined in terms of all of the alters for a particular ego. In the latter case, we could define a contagion effect that compares the mean counterfactual outcome for an ego had the mean outcome among the alters been set to one value as opposed to a different value. For simplicity, in the remaining sections we will talk about alter-ego pairs rather than clusters of an ego with all of its alters. This is in keeping with the existing applied literature, but it is important to note that close attention should be paid in future work to the definition of causal contagion effects for non-dyadic data. Numerous papers and researchers have addressed the definition of counterfactuals and causal effects in settings with interference (e.g. Aronow and Samii, 2012, Halloran and Struchiner, 1995, Halloran and Hudgens, 2011; Hong and Raudenbush 2006; Hudgens and Halloran 2008: Ogburn and VanderWeele, 2014a: Rosenbaum, 2007, Rubin, 1990; Sobel |2006; Tchetgen Tchetgen and VanderWeele, 2012); similar attention should be paid to contagion effects.

\section{Confounding}

Confounding, is, loosely, the presence of a non-causal association that may be misinterpreted as a causal effect of one variable on another. Most commonly, confounding is due to the presence of a confounder that has a causal effect on both the hypothesized cause and the hypothesized effect. Such a confounder generates an association between the hypothesized cause and effect which, without careful analysis, could be taken as evidence of a causal effect. There are two types of confounding that are nearly ubiquitous and especially intransigent in the context of contagion effects in social networks: homophily is the tendency of people who are similar to begin with to share network ties, and environmental confounding is the tendency of people who share network ties to also share environmental exposures that could jointly affect their outcomes. We elucidate these two types of confounding below. 


\subsection{Homophily}

Consider the Faber College student body. Suppose that two students, Pat and Lee, meet in September and bond over the fact that they both used to be competitive runners but recently developed injuries that prevent them from running and from participating in other active hobbies they used to enjoy. Soon Pat and Lee are close friends. Over the course of a few months, the sedentary lifestyle catches up with Pat, who gains a considerable amount of weight. It takes longer for Lee, but by the close of the school year Lee has also gained a lot of weight. If you did not have access to the back story and only observed that Pat gained weight and then Pat's close friend Lee did too, this looks like potential evidence of a causal effect of Pat's change in BMI on Lee's change in BMI. In fact, this is a case of homophily: unobserved covariates related to the propensity to gain weight (in this case, recent injury) caused Pat and Lee to become friends and also caused them to both undergo changes in BMI.

Some carefully considered studies attempt to control for all sources of homophily (see Shalizi and Thomas, 2011 for details and references), but this is generally not possible unless researchers have a high degree of control over data collection and can collect extremely rich (and therefore expensive!) data on the covariates that affect ties. Any traits that are related to the formation, duration, or strength of ties and to the outcome of interest must be measured. For some outcomes, such as infectious diseases, it may be possible to enumerate and observe all such traits, but for other outcomes, such as BMI, endless permutations of the Pat-and-Lee story are possible (e.g. friendship based on shared body norms, shared love of sugary snacks, shared appreciation for a particular celebrity whose BMI changes could affect both Pat and Lee's, etc.), making it nearly impossible to control for all potentially confounding traits. In addition to the challenge of enumerating the potentially confounding traits, there are huge costs to collecting such rich data, and available social network data are highly unlikely to include adequate covariates.

For these reasons, researchers have developed clever tricks to try to control for homophily using only data the network and the outcome of interest. One such trick is to include both the alter and the ego's outcomes ate time $t-2$ as covariates in a regression of the ego's outcome at time $t$ on the alter's outcome at time $t-1$. The argument used to justify this method is that any traits related to tie formation and to the outcome are fully captured by the similarity in the alter and ego's outcomes at time $t-2$; any association between the alter's outcome at time $t-1$ and the ego's at time $t$ after controlling for this baseline similarity must be due to contagion. But the story of Pat and Lee demonstrates one flaw in this argument: baseline traits can affect outcome trajectories over time and so conditioning on the outcome at a single time point does not render all future outcome measures independent of the baseline covariates. Another flaw in the argument is that homophily operates not only through the propensity to form ties, but also through the propensity to maintain ties and through the strength of the ties; neither strength nor duration can be captured by past outcomes (Noel and Nyhan, 2011). Furthermore, Shalizi and Thomas (2011) demonstrated that, even if a baseline trait only affects friendship formation (not 
strength or duration), merely conditioning on the presence of a tie, which is inherent in all analyses focused on alter-ego pairs, creates a spurious association between the alter's outcome at time $t-1$ and the ego's outcome at time $t$. This is because the presence of a tie is a collider: a common effect of two variables, conditioning on which creates a spurious association between the two causes. (For an accessible review of colliders see Elwert and Winship, 2014,)

Another clever trick is to compare the strength of the association between an alter's and an ego's outcomes across different types of ties: undirected, or mutual; directed, with the ego naming the alter as a friend but not vice versa; and directed, with the alter naming the ego as a friend but not vice versa. Suppose Pat claims Lee as a friend but Lee does not claim Pat as a friend. Any similarity in baseline traits that Pat and Lee share is a symmetric relationship, the argument goes, and therefore if the regression of Pat's BMI at time $t$ on Lee's BMI at time $t-1$ results in a larger coefficient than does the regression of Lee's BMI at time $t$ on Pat's BMI at time $t-1$, this is evidence of contagion. Unfortunately, this argument is also flawed (Lyons, 2011, Shalizi and Thomas, 2011). This is because, somewhat counterintuitively, similarity in baseline traits does not have to be symmetric. Suppose Pat claims Lee as a friend because Lee is the only person Pat knows who is going through a painful separation with running and other active hobbies, while Lee participates in a support group for recently injured former runners and considers only one participant, Lou, who has the exact same injury and prognosis, as a friend. By construction, even though Lee is the node with the most baseline similarity to Pat from among all of Pat's potential friends, the reverse is not true: Lou, not Pat, is the node with the most similarity to Lee from among all of Lee's potential friends. Therefore, if Lou's outcome at time $t-1$ has a stronger association with Lee's outcome at time $t-1$ than Pat's does, this could be evidence of greater similarity on baseline characteristics rather than contagion. Furthermore, it can be shown that a similar story results in reciprocated ties having the strongest association of all (Lyons, 2011). Shalizi and Thomas (2011) used a slightly different data-generating process to show that purported evidence for contagion due to asymmetry in the association of an alter's outcome with an ego's outcome for different types of ties is consistent with homophily rather than contagion.

\subsection{Shared environment}

Let's turn to a different pair of Faber students, Cam and Sam, who both decided to move off campus to a neighborhood across town from the college. Over the course of the school year, both the grocery store and the gym in their neighborhood closed down and were replaced with fast food restaurants. Cam immediately starts taking every meal at the fast food joint and gains weight fairly quickly, while Sam holds out for several months, taking the bus to a distant grocery store, but when time winter weather and final exams pile on Sam, too, falls prey to the fast food marketing. By the end of the year both students have gained weight. This is confounding due to 
shared environment, another source of confounding that plagues attempts to learn about contagion from observational data. People who share network ties tend to live near each other, work together, pay attention to the same information, or work in the same industry, all of which can generate confounding due to shared environment (which need not be restricted to physical environment). Note that confounding due to shared environment is present whether Cam and Sam are friends because they live in the same neighborhood or they moved to the same neighborhood because they were friends. The distinction between homophily and shared environment is not always clearcut; if Cam and Sam became friends because they lived in the same neighborhood that would simultaneously be an example of homophily and of shared environment. The same strategies described above for dealing with homophily have been used in an attempt to control for confounding due to shared environment, but similar reasoning controverts their effectiveness.

Cohen-Cole and Fletcher (2008) proposed controlling for confounding by shared environment by including fixed effects for "community" in regressions of an ego's outcome at time $t$ on an alter's outcome at time $t-1$. If all such confounding occurs due to clearly delineated and known communities, like well-defined neighborhoods in the example above, this is potentially a good solution, though in many cases the operative communities, or their membership, will likely be unknown.

\section{Dependence}

Suppose confounding is not an issue, because researchers at Faber were well-funded and prescient enough to collect data on every possible confounder of the contagion effect, and further suppose that the researchers have a model-maybe a regression, maybe a propensity-score based method (Aral et al 2009), maybe some other model-that they believe gives an estimate of the causal contagion effect. We now turn to the question of how to perform valid statistical inference using a model fit to data from a social network. The issue of valid statistical inference is entirely separate from the issue of confounding or even contagion; it applies whether we want to estimate a simple mean or a complicated causal effect. The key points made in this section apply to anything that we want to estimate using social network data. Most estimators of causal effects, including The coefficient on the alter's outcome at time $t-1$ in a regression of the ego's outcome at time $t$, are closely related to sample means (to be technical, they are M-estimators), so all of the points made below apply.

Going back to Faber College, administrators are now interested in the simpler problem of estimating the mean BMI for the student body at the end of the school year. There are $n$ students, or nodes in the social network comprised of students, and each one furnishes an observed BMI measurement $Y_{i}$. Our goal is to perform valid (frequentist) statistical inference about the true mean $\mu$ of $Y$ using a sample mean $\bar{Y}=\frac{1}{n} \sum_{i=1}^{n} Y_{i}$ of dependent observations $\mathbf{Y}=\left(Y_{1}, \ldots, Y_{n}\right)$, where the dependence among observations is determined or informed by network structure. But for 
the dependence, this is a familiar problem. In general, when we want to use a sample mean to perform inference about a true mean, we take the sample mean as our point estimate, calculate a standard error for the sample mean, and tack on a confidence interval based on that standard error. The unique challenge for the social network setting is the effect of dependence on the standard error. To keep things as simple as possible, let's assume that $Y_{i}, \ldots, Y_{n}$ are identically, though not independently, distributed, so the mean of $Y_{i}$ is $\mu$ and the variance of $Y_{i}$ is $\sigma^{2}$, which we assume is finite, for all $i$. (In fact, it is easier to deal with observations that are not identically distributed than it is to deal with observations that are dependent, so relaxing this assumption is not too difficult.)

Recall that the standard error of $\bar{Y}$ is the square-root of its variance, where

$$
\begin{array}{r}
\operatorname{Var}(\bar{Y})=\frac{1}{n^{2}} \operatorname{Var}\left(\sum_{i=1}^{n} Y_{i}\right) \\
=\frac{1}{n^{2}}\left\{\sum_{i=1}^{n} \sigma^{2}+\sum_{i \neq j} \operatorname{cov}\left(Y_{i}, Y_{j}\right)\right\} \\
=\frac{\sigma^{2}}{n}+\frac{1}{n^{2}} \sum_{i \neq j} \operatorname{cov}\left(Y_{i}, Y_{j}\right) .
\end{array}
$$

When $Y_{i}, \ldots, Y_{n}$ are independent, the covariance term $\operatorname{cov}\left(Y_{i}, Y_{j}\right)$ is equal to 0 for all $i \neq j$ pairs, so the variance of $\bar{Y}$ is $\frac{\sigma^{2}}{n}$, which should be familiar from any introductory statistics or data analysis class. But when $Y_{i}, \ldots, Y_{n}$ are dependent, in particular when they are positively correlated (which is the type of dependence that we would expect to see in just about every social network setting), the variance of $\bar{Y}$ is bigger than $\frac{\sigma^{2}}{n}$ because it includes the term $\frac{1}{n^{2}} \sum_{i \neq j} \operatorname{cov}\left(Y_{i}, Y_{j}\right)$. Define $b_{n}=\frac{1}{n} \sum_{i \neq j} \operatorname{cov}\left(Y_{i}, Y_{j}\right)$. Then

$$
\operatorname{var}(\bar{Y})=\frac{\sigma^{2}}{n /\left(1+\frac{b_{n}}{\sigma^{2}}\right)}
$$

and we can see that the factor by which the variance of $\bar{Y}$ is bigger than what it would be if $Y_{i}, \ldots, Y_{n}$ were independent is $\left(1+\frac{b_{n}}{\sigma^{2}}\right)$. We call $n /\left(1+\frac{b_{n}}{\sigma^{2}}\right)$ the effective sample size of our sample of $n$ dependent observations $Y_{1}, \ldots, Y_{n}$. The effective sample size $n /\left(1+\frac{b_{n}}{\sigma^{2}}\right)$ is smaller than the true sample size $n$; heuristically this is because each observation $Y_{i}$ contains some new information about the target of inference $\mu$ and some information that is rendered redundant by dependence. Under independence each observation furnishes 1 "bit" of information about $\mu$, whereas under dependence each observation furnishes only $1 /\left(1+\frac{b_{n}}{\sigma^{2}}\right)$ bit of information about $\mu$.

In order to explain the impact of this dependence on statistical inference, we first review the standard inferential procedure for independent data. When $Y_{i}, \ldots, Y_{n}$ are independent, a typical procedure would be to calculate an approximate $95 \%$ 
confidence interval for $\mu$ as $\bar{Y} \pm 1.96 \times \frac{\hat{\sigma}}{\sqrt{n}}$, where $\hat{\sigma}$ is the square root of an estimate of the variance of $Y$. The factor 1.96 is the 97.5th quantile of the standard Normal distribution; t-distribution quantiles could be used instead to account for the fact that $\sigma$ is estimated rather than known. This procedure relies on several preliminaries: (1) $\bar{Y}$ is unbiased for $\mu$, (2) $\bar{Y}$ is approximately Normally distributed, and (3) $\frac{\hat{\sigma}}{\sqrt{n}}$ is a good estimate of the variance of $\bar{Y}$. These preliminaries hold, at least approximately, in most settings with independent data and moderate to large $n$. Dependence doesn't affect (1), but it does affect (2) and (3).

When $Y_{i}, \ldots, Y_{n}$ are independent, the Central Limit Theorem (CLT) tells us that $\sqrt{n}(\bar{Y}-\mu)$ converges in distribution to a Normal distribution as $n \rightarrow \infty$. The factor $\sqrt{n}$ is called the rate of convergence and it is needed to make sure that the variance of $\sqrt{n}(\bar{Y}-\mu)$ is not 0 , in which case $\sqrt{n}(\bar{Y}-\mu)$ would converge to a constant rather than a distribution, and is not infinite, in which case $\sqrt{n}(\bar{Y}-\mu)$ would not converge at all. The variance of $\bar{Y}$ (equivalently, the variance of $\bar{Y}-\mu$ ) is $\sigma^{2} / n$, so the variance of $\sqrt{n}(\bar{Y}-\mu)$ is $n \times\left(\sigma^{2} / n\right)=\sigma^{2}$, which is a positive, finite constant. When $Y_{i}, \ldots, Y_{n}$ are dependent, the rate of convergence may be different (slower) than $\sqrt{n}$. (In fact, if the dependence is strong and widespread enough, the CLT may not hold at all; determining what types of social network dependence are consistent with the CLT is an important area for future study.) This is because the rate of convergence is determined by the effective sample size instead of by $n$ : the variance of $\bar{Y}$ is $\sigma^{2} /\left\{n /\left(1+\frac{b_{n}}{\sigma^{2}}\right)\right\}$, so (as long as a CLT holds), $\sqrt{n /\left(1+\frac{b_{n}}{\sigma^{2}}\right)}(\bar{Y}-\mu)$ will converge to a Normal distribution as $n \rightarrow \infty$ and the rate of convergence is given by $\sqrt{n /\left(1+\frac{b_{n}}{\sigma^{2}}\right)}$ rather than $\sqrt{n}$. Sometimes, in particular when $b_{n}$ is fixed as $n \rightarrow \infty$, this distinction will be meaningless. But sometimes, when $b_{n}$ grows with $n$, it is a meaningfully slower rate of convergence. (Note that $b_{n} / n$ must converge to 0 as $n \rightarrow \infty$ in order for a CLT to hold, so $b_{n}$ must grow slower than $n$.) This matters because it informs when the approximate Normality of the CLT kicks in, i.e. at what sample size it is safe to assume that $\bar{Y}$ is approximately Normally distributed. Many different rules of thumb exist for determining when approximate Normality holds; one popular rule of thumb is that $n=30$ suffices. With dependent data, this number is larger, and sometimes considerably so. The effective sample size, rather than $n$, should be used to assess whether the sample size is large enough to approximate the distribution of $\bar{Y}$ with a Normal distribution. When researchers ignore dependence and rely on the Normal approximation in samples that have large enough $n$ but not large enough effective sample size, there is no reason to think that their $95 \%$ confidence intervals will have good coverage properties.

Ignoring dependence is most dangerous when estimating the standard error of $\bar{Y}$. Any estimate of $\operatorname{var}(\bar{Y})$ that is based only on the marginal variances $\sigma^{2}$ of $Y_{i}$ and ignore the covariances $\operatorname{cov}\left(Y_{i}, Y_{j}\right)$ will underestimate the standard error of $\bar{Y}$, often severely. Inference that is based on an underestimated standard error is anticonservative: confidence intervals are narrower than they should be and p-values are lower than they should be, leading researchers to draw conclusions that are not in fact substantiated by the data. Even if each observation is dependent only on a 
fixed and finite number of other observations, so that dependence is asymptotically negligible and does not affect the rate of convergence of the CLT, in finite samples ignoring the covariance terms in $\operatorname{var}(\bar{Y})$ could still have substantial implications on inference. This is particularly a problem because no good solutions exist. Statisticians are good at dealing with dependence that arises due to space or time, or even other more complicated processes that can be expressed using Euclidean geometry. But dependence that is informed by a network is very different from these well-understood types of dependence, and, unfortunately, statisticians are only just beginning to develop methods for taking it into account. Most published research about social contagion uses regression models or generalized estimating equations (GEEs) to estimate contagion effects; though some of these models account for the dependence due to observing the same nodes over multiple time points, none of them account for dependence among nodes.

\subsection{Sources of network dependence}

In the literature on spatial and temporal dependence, dependence is often implicitly assumed to be the result of latent traits that are more similar for observations that are close in Euclidean distance than for distant observations. This type of dependence is likely to be present in many network contexts as well. In networks, edges present opportunities to transmit traits or information, and contagion or influence is an important additional source of dependence that depends on the underlying network structure.

Latent trait dependence will be present in data sampled from a network whenever observations from nodes that are close to one another are more likely to share unmeasured traits than are observations from distant nodes. Homophily is a paradigmatic example of latent trait dependence. If the outcome under study in a social network has a genetic component, then we would expect latent variable dependence due the fact that family members, who share latent genetic traits, are more likely to be close in social distance than people who are unrelated. If the outcome were affected by geography or physical environment, latent variable dependence could arise because people who live close to one another are more likely to be friends than those who are geographically distant. Of course, whether these traits are latent or observed they can create dependence, but if they are observed then conditioning on them renders observations independent, so only when they are latent do they result in dependence that requires new tools for statistical inference. Just like in the spatial dependence context, there is often little reason to think that we could identify, let alone measure, all of these sources of dependence. The notions of latent sources of homophily or latent correlates of shared environment are familiar from the discussion of confounding, above, but there is an important distinction to be made between latent sources of confounding and latent sources of dependence: in order to be a source of unmeasured confounding, a latent trait must affect both the exposure (e.g. the alter's outcome at time $t-1$ ) and the outcome (ego's outcome at time $t$ ) 
of interest. In order to be a source of dependence, a latent trait must affect two or more outcomes of interest. Latent trait dependence is the most general form of dependence, in that it provides no structure that can be harnessed to propel inference. In order to make any progress towards valid inference in the presence of latent trait dependence, some structure must be assumed, namely that the range of influence of the latent traits is primarily local in the network and that any long-range effects are negligible.

Contagion or influence arises when the outcome under study is transmitted from node to node along edges in the network. The diagram in Figure 1 depicts contagion in a network with three nodes in which node 2 is connected to nodes 1 and 3 but there is no edge between 1 and 3. $Y_{i}^{t}$ represents the outcome for node $i$ at time $t$, and the unit of time is small enough that at most one transmission event can occur between consecutive time points. Dependence due to contagion has known, though possibly unobserved, structures that can sometimes be harnessed to facilitate inference; we touch on this briefly in Section 6 Crucially, whenever contagion is present so is dependence, and therefore statistical analysis must take dependence into account in order to result in valid inference.

Fig. 1 Dependence by contagion

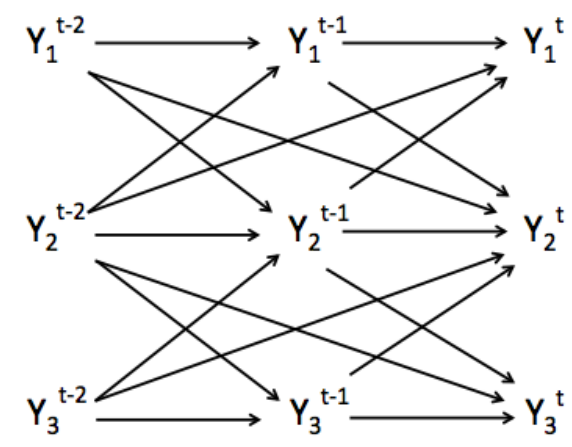

\section{Solutions}

Researchers have known for decades that learning about contagion from observational data is fraught with difficulty, perhaps most famously expressed by Manski (1993). Recent years have seen incremental methodological progress, but huge hurdles remain. Most of the constructive ideas in Shalizi and Thomas (2011) involve bounding contagion effects rather than attempting to point identify them; looking for bounds rather than point estimates is a general approach that could prove fruitful in the future. Indeed, Ver Steeg and Galstyan (2010) built upon the ideas in 
Shalizi and Thomas (2011) and were able to derive bounds on the association due to homophily on traits that do not change over time ("static homophily"). Another general approach is to make use of sensitivity analyses whenever an estimation procedure relies on assumptions that may not be realistic (e.g. VanderWeele, 2011). Some of the problems discussed above have solutions in some settings; below we discuss solutions that exploit features of specific settings rather than providing general approaches to the problem of estimating contagion effects. (Some of the material below was first published in Ogburn and Volfovsky, 2016,)

\subsection{Randomization}

If it is possible to randomize some members of a social network to receive an intervention, and if it is known that an alter's receiving an intervention can only affect the ego's outcome through contagion (as opposed to directly; see Ogburn and VanderWeele 2014a for discussion), then problems of confounding and dependence can be entirely obviated. Randomization-based inference, pioneered by Fisher (Fisher, 1922) and applied to network-like settings by Rosenbaum (2007) and Bowers et al (2013), is founded on the very intuitive notion that, under the null hypothesis of no effect of treatment on any subject (sometimes called the sharp null hypothesis to distinguish it from other null hypotheses that may be of interest), the treated and control groups are random samples from the same underlying distribution. Randomizationbased inference treats outcomes as fixed and treatment assignments as random variables: quantities that depend on the vector of treatment assignments are the only random variables in this paradigm. Therefore, dependence among outcomes is a non-issue. Typically this type of inference is reserved for hypothesis testing, though researchers have extended it to estimation. We leave the details, including several subtleties and challenges that are specific to the social network context, to a later chapter (see also Ogburn and Volfovsky, 2016 for a review).

Randomizing the formation of network ties themselves obviates confounding due to the effects of homophily on tie formation. A number of studies have taken advantage of naturally occurring randomizations of this kind, such as the assignment of students to dorm rooms (Sacerdote, 2000) or of children to classrooms (Kang, 2007). However, this does not suffice to control for the effects of homophily on tie strength or duration, or to control for confounding due to shared environment.

\subsection{Parametric models}

If researchers are willing to commit to certain types of parametric models, it may be possible isolate contagion from confounding (Snijders et al, 2007). It is a reliance on strong parametric models, for example, that underpins mathematical modeling 
or agent based modeling approaches to contagion (Burk et al, 2007; Snijders et al, 2010, Railsback and Grimm, 2011).

This might seem benign-after all, most statistical analyses rely on parametric models of one kind or another-but there is a fundamental difference between, for example, using a linear regression when the true underlying relationships is not linear, and relying on parametric models to identify a causal effect that is otherwise hopelessly confounded. In the first case, a misspecified model may bias the estimate we are interested in, often in ways that are well-understood, and often in proportion to the fit of the model to the data (i.e. the worse the misspecification, the greater the bias). In the latter case, at least in the absence of a model-specific proof otherwise, any hint of misspecification undermines the causal interpretation we would like to be able to justify and what looks like evidence of a causal effect could just be evidence of confounding. George Box's oft-cited aphorism, "all models are wrong but some are useful," justifies the use of misspecified parametric models in many settings, but when the parametric form of the model is the only bulwark against confounding, the model must (in the absence of a proof to the contrary) in fact be correct in order to be useful.

\subsection{Instrumental variable methods}

O'Malley et al (2014) proposed an instrumental variable (IV) solution to the problem of disentangling contagion from homophily. An instrument is a random variable, $V$, that affects exposure but has no effect on the outcome conditional on exposure. When the exposure - outcome relation suffers from unmeasured confounding but an instrument can be found that is not confounded with the outcome, IV methods can be used to recover valid estimates of the causal effect of the exposure on the outcome. In this case there is unmeasured confounding of the relation between an alter's outcome at time $t-1$ and an ego's outcome at time $t$ whenever there is homophily on unmeasured traits. Angrist and Pischke (2008), Greenland (2000), and Pearl (2000) provide accessible reviews of IV methods.

O'Malley et al (2014) propose using a gene that is known to be associated with the outcome of interest as an instrument. In their paper they focus on perhaps the most highly publicized claim of peer effects, namely that there are significant peer effects of body mass index (BMI) and obesity (Christakis and Fowler, 2007). If there is a gene that affects BMI but that does not affect other homophilous traits, then that gene is a valid instrument for the effect of an alter's BMI on his ego's BMI. The gene affects the ego's BMI only through the alter's manifest BMI (and it is independent of the ego's BMI conditional on the alter's BMI), and there is unlikely to be any confounding, measured or unmeasured, of the relation between an alter's gene and the ego's BMI.

There are two important challenges to this approach. First, the power to detect peer effects is dependent in part upon the strength of the instrument - exposure relation which, for genetic instruments, is often weak. Indeed, O'Malley et al (2014) 
reported low power for their data analyses. Second, in order to assess contagion at more than a single time point (i.e. the average effect of the alter's outcomes on the ego's outcomes up to that time point), multiple instruments are required. O'Malley et al (2014) suggest using a single gene interacted with age to capture time-varying gene expression, but this could further attenuate the instrument - exposure relation and this method is not valid unless the effect of the gene on the outcome really does vary with time; if the gene-by-age interactions are highly collinear then they will fail to act as differentiated instruments for different time points.

\subsection{Data from multiple independent networks}

When multiple independent networks are observed, the problems of confounding due to shared environment and of dependence may be considerably easier to deal with. A large literature on interference in causal inference is dedicated to inference in the setting where independent groups of individuals interact and affect one another within, but not between, groups; this is analogous to multiple independent social networks (see, e.g., Sobel, 2006; Hong and Raudenbush, 2006; Hudgens and Halloran 2008; Tchetgen Tchetgen and VanderWeele, 2012; Liu and Hudgens, 2014). If environmental factors can shared within but not across networks, it may be possible to control for confounding by shared environment via a fixed effect for each network, as in Cohen-Cole and Fletcher (2008).

\subsection{Contagion operating alone}

If researchers have reason to believe that there is no unmeasured homophily or features of shared environments that contribute to confounding or to dependence, i.e. if contagion is the only mechanism giving rise to either dependence or to associations among the outcomes of interest, then there are a few recent methodological advances that can be used to estimate contagion effects (van der Laan, 2012, Ogburn and VanderWeele, 2014b; Ogburn et al, 2017). Dependence due to contagion has known, though possibly unobserved, structures that can sometimes be harnessed to facilitate inference. Time and distance act as information barriers for dependence due to contagion, giving rise to many conditional independencies that can sometimes be used to make network dependence tractable. Two examples of the many conditional independencies that hold in Figure (1) are $\left[Y_{1}^{t} \perp Y_{2}^{t} \mid Y_{1}^{t-2}, Y_{2}^{t-2}, Y_{1}^{t-1}, Y_{2}^{t-1}\right]$ and $\left[Y_{1}^{t-1} \perp Y_{3}^{t} \mid Y_{2}^{t-2}\right]$. The first conditional independence statement illustrates the principle that outcomes measured at a particular time point are mutually independent conditional on all past outcomes. The second conditional independence statement illustrates the fact that outcomes sampled from two nonadjacent nodes are independent if the amount of time that passed between the two measurements was not sufficiently long for information to travel along the shortest path from one node 
to the other, conditional any information that could have simultaneously influenced the sampled nodes (in this case $Y_{2}^{t-2}$ ). Observing outcomes in a network on a fine enough time scale to observe all transmissions requires a richness of data that will not usually be available, and if the network under a contagious process is observed at a single time point, dependence due to contagion is indistinguishable from latent variable dependence and the structure is lost.

Acknowledgements This work was funded by the Office of Naval Research grant N00014-15-12343.

\section{References}

Ali MM, Dwyer DS (2009) Estimating peer effects in adolescent smoking behavior: A longitudinal analysis. Journal of Adolescent Health 45(4):402-408

Angrist JD, Pischke JS (2008) Mostly harmless econometrics: An empiricist's companion. Princeton university press

Aral S, Muchnik L, Sundararajan A (2009) Distinguishing influence-based contagion from homophily-driven diffusion in dynamic networks. Proceedings of the National Academy of Sciences 106(51):21,544-21,549

Aronow PM, Samii C (2012) Estimating average causal effects under general interference. Tech. rep.

Besag J (1974) On spatial-temporal models and markov fields. In: Transactions of the Seventh Prague Conference on Information Theory, Statistical Decision Functions, and Random Processes, Springer, pp 47-55

Bowers J, Fredrickson MM, Panagopoulos C (2013) Reasoning about interference between units: A general framework. Political Analysis 21(1):97-124

Burk WJ, Steglich CE, Snijders TA (2007) Beyond dyadic interdependence: Actororiented models for co-evolving social networks and individual behaviors. International journal of behavioral development 31(4):397-404

Cacioppo JT, Fowler JH, Christakis NA (2009) Alone in the crowd: the structure and spread of loneliness in a large social network. Journal of personality and social psychology 97(6):977

Christakis N, Fowler J (2007) The spread of obesity in a large social network over 32 years. New England journal of medicine 357(4):370-379

Christakis N, Fowler J (2008) The collective dynamics of smoking in a large social network. New England journal of medicine 358(21):2249-2258

Christakis N, Fowler J (2010) Social network sensors for early detection of contagious outbreaks. PloS one 5(9):e12,948

Cohen-Cole E, Fletcher JM (2008) Is obesity contagious? social networks vs. environmental factors in the obesity epidemic. Journal of Health Economics 27(5):1382-1387

Elwert F, Winship C (2014) Endogenous selection bias: The problem of conditioning on a collider variable. Annual Review of Sociology 40:31-53 
Fisher RA (1922) On the mathematical foundations of theoretical statistics. Philosophical Transactions of the Royal Society of London Series A, Containing Papers of a Mathematical or Physical Character 222:309-368

Goetzke F (2008) Network effects in public transit use: evidence from a spatially autoregressive mode choice model for new york. Urban Studies 45(2):407-417

Greenland S (2000) An introduction to instrumental variables for epidemiologists. International journal of epidemiology 29(4):722-729

Halloran M, Hudgens M (2011) Causal inference for vaccine effects on infectiousness. The University of North Carolina at Chapel Hill Department of Biostatistics Technical Report Series p 20

Halloran ME, Struchiner CJ (1995) Causal inference in infectious diseases. Epidemiology 6(2):142-151

Hernán MA (2004) A definition of causal effect for epidemiological research. Journal of Epidemiology and Community Health 58(4):265-271

Hong G, Raudenbush S (2006) Evaluating kindergarten retention policy. Journal of the American Statistical Association 101(475):901-910

Hudgens M, Halloran M (2008) Toward causal inference with interference. Journal of the American Statistical Association 103(482):832-842

Kang C (2007) Classroom peer effects and academic achievement: Quasirandomization evidence from south korea. Journal of Urban Economics 61(3):458-495

van der Laan MJ (2012) Causal inference for networks. UC Berkeley Division of Biostatistics Working Paper Series Working Paper 300

Lauritzen SL, Richardson TS (2002) Chain graph models and their causal interpretations. Journal of the Royal Statistical Society: Series B (Statistical Methodology) 64(3):321-348

Lazer D, Rubineau B, Chetkovich C, Katz N, Neblo M (2010) The coevolution of networks and political attitudes. Political Communication 27(3):248-274

Lee LF (2004) Asymptotic distributions of quasi-maximum likelihood estimators for spatial autoregressive models. Econometrica 72(6):1899-1925

Lin X (2005) Peer effects and student academic achievement: an application of spatial autoregressive model with group unobservables. Unpublished manuscript, Ohio State University

Liu L, Hudgens MG (2014) Large sample randomization inference of causal effects in the presence of interference. Journal of the american statistical association 109(505):288-301

Lyons R (2011) The spread of evidence-poor medicine via flawed social-network analysis. Statistics, Politics, and Policy 2(1)

Manski CF (1993) Identification of endogenous social effects: The reflection problem. The review of economic studies 60(3):531-542

Noel H, Nyhan B (2011) The unfriending problem: The consequences of homophily in friendship retention for causal estimates of social influence. Social Networks 33(3):211-218

Ogburn EL, VanderWeele TJ (2014a) Causal diagrams for interference. Statistical Science 
Ogburn EL, VanderWeele TJ (2014b) Vaccines, contagion, and social networks. arXiv preprint arXiv: 14031241

Ogburn EL, Volfovsky A (2016) Networks. In: P B, P D, M K, van der Laan MJ (eds) Handbook of Big Data, Chapman \& Hall/CRC

Ogburn EL, O S, van der Laan MJ, I D (2017) Causal inference for social network data with contagion. Tech. rep., Johns Hopkins University

O'Malley AJ, Elwert F, Rosenquist JN, Zaslavsky AM, Christakis NA (2014) Estimating peer effects in longitudinal dyadic data using instrumental variables. Biometrics

O'Malley JA, Marsden PV (2008) The analysis of social networks. Health services and outcomes research methodology 8(4):222-269

Pearl J (2000) Causality: models, reasoning and inference. Cambridge Univ Press

Railsback SF, Grimm V (2011) Agent-based and individual-based modeling: a practical introduction. Princeton university press

Rosenbaum P (2007) Interference between units in randomized experiments. Journal of the American Statistical Association 102(477):191-200

Rosenquist JN, Murabito J, Fowler JH, Christakis NA (2010) The spread of alcohol consumption behavior in a large social network. Annals of Internal Medicine 152(7):426-433

Rubin D (1990) On the application of probability theory to agricultural experiments. essay on principles. section 9. comment: Neyman (1923) and causal inference in experiments and observational studies. Statistical Science 5(4):472-480

Rubin DB (2005) Causal inference using potential outcomes: Design, modeling, decisions. Journal of the American Statistical Association 100(469):322-331

Sacerdote B (2000) Peer effects with random assignment: Results for dartmouth roommates. Tech. rep., National Bureau of Economic Research

Shalizi CR (2012) Comment on "why and when 'flawed' social network analyses still yield valid tests of no contagion". Statistics, Politics, and Policy 3(1)

Shalizi CR, Thomas AC (2011) Homophily and contagion are generically confounded in observational social network studies. Sociological Methods \& Research 40(2):211-239

Snijders T, Steglich C, Schweinberger M (2007) Modeling the coevolution of networks and behavior. na

Snijders TA, Van de Bunt GG, Steglich CE (2010) Introduction to stochastic actorbased models for network dynamics. Social networks 32(1):44-60

Sobel M (2006) What do randomized studies of housing mobility demonstrate? Journal of the American Statistical Association 101(476):1398-1407

Tchetgen Tchetgen EJ, VanderWeele T (2012) On causal inference in the presence of interference. Statistical Methods in Medical Research 21(1):55-75

Thomas A (2013) The social contagion hypothesis: comment on 'social contagion theory: examining dynamic social networks and human behavior'. Statistical in Medicine 32(4):581-590

VanderWeele TJ (2011) Sensitivity analysis for contagion effects in social networks. Sociological Methods \& Research 40(2):240-255 
VanderWeele TJ, Ogburn EL, Tchetgen EJT (2012) Statistics, politics, and policy. Politics, and Policy 3(1):4

Ver Steeg G, Galstyan A (2010) Ruling out latent homophily in social networks. NIPSWorkshop on Social Computing 